\title{
Efeito das atividades antrópicas na cobertura vegetal na Reserva Florestal de Mecubúri, Moçambique
}

\author{
Aguinaldo Joaquim MÁRIO1, Sailon Augusto Roia MESA²-IM, \\ Caetano Miguel Lemos SERROTE ${ }^{3 *}$
}

\author{
${ }^{1} \mathrm{AJ}$ Consultoria Florestal, Mocuba, Moçambique. \\ 2Instituto de Investigação Agrária de Moçambique, Nampula, Moçambique. (In Memorian) \\ ${ }^{3}$ Universidade Lúrio, Faculdade de Ciências Agrárias, Unango, Moçambique \\ *E-mail: serrotec@yahoo.com.br \\ (ORCID: 0000-0001-6613-4050; (In Memorian); 0000-0002-0275-2201)
}

Recebido em 14/11/2021; Aceito em 17/12/2021; Publicado em 28/12/2021.

\begin{abstract}
RESUMO: O estudo foi realizado na Reserva Florestal de Mecubúri, em Moçambiquee, visando avaliar os efeitos das atividades antrópicas sobre a cobertura florestal. Áreas de queimada, agrícola e de exploração florestal foram comparadas com uma área controle em termos de composição e fitossociologia. Em cada área, foram estabelecidas, de maneira sistemática, parcelas quadrangulares de $20 \times 20 \mathrm{~m}$, distantes $20 \mathrm{~m}$ uma da outra, sendo realizado o levantamento da vegetação adulta e da regeneração. Foram determinados índices de similaridade, diversidade e riqueza. Os resultados indicaram existir dissimilaridade entre as áreas, sendo que as espécies Julbernardia globiflora, Diplorbynchus condylocarpon, Brachystegia utilis, Millettia stublmannii, Afzelia quanzensis, Burkea africana e Cussonia arborea destacaram-se em termos de abundância, frequência, dominância, Índice de Valor de Importância (IVI) e regeneração. O maior número de árvores por hectare foi encontrado na área de controle e o menor número foi encontrado na área agrícola. Com base no índice de dispersão de Morisita, a maior parte das espécies distribui-se de forma aleatória e agrupada. A distribuição diamétrica das árvores seguiu o padrão de J-invertido, característico de florestas naturais. As práticas de queimadas, agricultura e exploração florestal contribuem na redução da cobertura florestal na Reserva Florestal de Mecubúri.
\end{abstract}

Palavras-chave: fitossociologia; conservação; desmatamento.

\section{The effect of anthropic activities on forest coverage in the Mecubúri Forest Reserve, Mozambique}

\begin{abstract}
The study was carried out in the Mecubúri Forest Reserve, Mozambique, with the purpose of evaluating the effects of human activities on the forest cover. Burning, agricultural and logging areas were compared with a control area in terms of composition and phytosociology. In each area were systematically established $20 \times 20 \mathrm{~m}$ quadrangular plots, $20 \mathrm{~m}$ apart from each other, in which were carried out the survey of adult vegetation and regeneration. The similarity, diversity and richness indices were determined. The results showed that there is dissimilarity between the areas and the species Julbernardia globiflora, Diplorbynchus condylocarpon, Brachystegia utilis, Millettia stublmannii, Afzelia quanzensis, Burkea africana e Cussonia arborea stood out in terms of abundance, frequency, dominance, Importance Value Index (IVI) and regeneration. The largest number of trees per hectare was found in the control area, and the smallest number was found in the agricultural area. Based on the Morisita dispersion index, most species have random and clustered distribution. The diametric structure of the trees followed the inverted-j, typical of natural forests. Burning, agriculture and logging contribute to the reduction of forest cover in the Mecubúri Forest Reserve.
\end{abstract}

Keywords: phytosociology; conservation; deforestation.

\section{INTRODUÇÃO}

Moçambique localiza-se na Costa Oriental da África Austral e possui uma área de aproximadamente 78.475 .500 ha. É um dos poucos países que ainda mantem uma área considerável de florestas nativas e com grande diversidade de espécies, distribuídas em cerca de 40,6 milhões de hectares, que perfazem cerca de $51 \%$ do território nacional (MARZOLI, 2007). As florestas produtivas cobrem cerca de 26,7 milhões de hectares, sendo que treze milhões de hectares não são favoráveis para a produção madeireira e a maior parte localiza-se dentro de áreas de conservação (Parques Nacionais, Reservas Florestais e outras). As áreas de conservação albergam uma diversidade de recursos que fornecem bens e serviços, dos quais as populações dependem, como recursos alimentares, medicinais, econômicos e ambientais. Apesar disso, nessas áreas têm-se praticado diversas atividades como a agricultura itinerante $\mathrm{e}$ caça, caracterizadas pelo uso do fogo, na maioria das vezes não controlado e que resulta na degradação ambiental (NUBE et al., 2016).

Souza et al. (2001) sustentam que as atividades antrópicas têm comprometido a sustentabilidade dos ecossistemas florestais, por causar impactos negativos que contribuem de forma significativa para a perda de espécies florestais e faunísticas, destruição de habitats, fragmentos de paisagem, 
exposições do solo a intempéries, poluição da água, dentre outros.

Mecubúri é um dos poucos distritos da Província de Nampula, no norte de Moçambique, que possui uma das maiores Reservas Florestais do país, que abriga considerável número de espécies florestais e faunísticas, e no seu interior encontram-se assentamentos humanos.

O presente trabalho teve como propósito avaliar os efeitos das atividades antrópicas sobre a cobertura florestal da Reserva de Mecubúri, com base na análise da composição e distribuição espacial de espécies florestais.

\section{MATERIAL E MÉTODOS}

\section{1. Área de estudo}

A Reserva Florestal de Mecubúri (RFM) é considerada a maior Reserva Florestal do país, cobrindo uma área total de 230.000ha correspondente a um triângulo entre as localidades de Mecubúri - sede, Muite e Imala (MANANZE et al., 2016). Foi criada pela Portaria número 8459 - Boletim Oficial no 29, I Série, de 22 de julho de 1950, com o principal objetivo de impedir o avanço da agricultura e conservar o ecossistema, garantindo assim a protecção da bacia hidrográfica do Rio Mecubúri (SITOE; MAÚSSE-SITOE, 2009).



Figura 1. Localização da área de estudo.

Figure 1. Location of the study area.

Nas encostas superiores existem solos vermelhos e ou alaranjados. O clima é predominantemente quente, oceânico, seco (na zona norte da região) a chuvoso (no restante). A precipitação média anual varia entre 500 a $1200 \mathrm{~mm}$ podendo exceder por vezes para $1500 \mathrm{~mm}$ tornando-se um clima subúmido chuvoso. A temperatura média anual é de $25^{\circ} \mathrm{C}$, sendo janeiro e fevereiro os meses mais quentes (com 26,6 ${ }^{\circ} \mathrm{C}$ e $25,8^{\circ} \mathrm{C}$, respectivamente) e julho o mês mais frio e seco $\left(\operatorname{com} 21,2^{\circ} \mathrm{C}\right)$. A reserva é atravessada por vários rios, permanentes e sazonais, sendo o rio Mecubúri o mais importante (MANANZE et al., 2016).

A RFM apresenta grande diversidade de espécies faunísticas, destacando-se Elefante-africano (Loxodonta africana), Kudu (Tragelaphus strepsiceros), Imbabala (Tragelaphus scriptus), Piva (Kobusellipsi prymus), Chango (Redunca arundinum), Cabrito - cinzento (Sylvicar pagrimmia), Leopardo (Panthera pardus), Facochero (Phacochoerus aethipicus) e o Leão (Panthera leo), para além de inúmeras espécies de insetos, répteis, pássaros e pequenos roedores (MANANZE et al., 2016).

O Miombo é o principal tipo de vegetação da Reserva Florestal de Mecubúri, sendo caracterizado pela dominância de uma ou mais espécies do género Brachystegia e Julbernardia, e da espécie Isoberlina angolensis (Fabaceae, Subfamília Caesalpinioideae) na região. Ao longo do rio Mecubúri encontram-se florestas de galeria, com dominância de espécies como Spirostachys africana, Schinziophyton rautaneniie, Milletia stublmanni e ainda pequenas árvores como Friesodielsia obovatae e várias espécies do género Strychnus.

O distrito de Mecubúri apresenta uma população estimada em 142.687 habitantes, com uma densidade populacional de $19,7 \mathrm{hab} / \mathrm{km}^{2}$. Estima-se que vivam na área da Reserva Florestal de Mecubúri cerca de 40 mil pessoas integradas em 12 mil agregados familiares e distribuídos por 15 regulados. A maior parte dessa população (81\%) é 
analfabeta, sobretudo as mulheres, sendo a agricultura familiar a base de subsistência das famílias (MAE, 2005).

\subsection{Coleta e processamento de dados}

Para a identificação das atividades desenvolvidas na RFM recorreu-se à entrevistas semiestruturadas e a observação direta. A seleção dos indivíduos para a entrevista foi feita mediante a amostragem não probabilística (amostragem bola de neve).

Para comparar a composição florística e fitossociologia foram selecionadas três áreas em função da atividade praticada, nomeadamente, áreas de queimadas, de prática agrícola ou de exploração florestal e uma área de controle (sem intervenção humana). Foram estabelecidas parcelas amostrais sistemáticas de $20 \times 20 \mathrm{~m}$, distantes $20 \mathrm{~m}$ uma da outra, totalizando 13 parcelas/ha. Nessas parcelas foram realizados o levantamento das espécies, análise da composição e determinados os parâmetros fitossociológicos (abundância, frequência, dominância e índice de valor de importância) de todos os indivíduos com DAP superior a $0,10 \mathrm{~m}$. No interior de cada parcela foram estabelecidas quatro subparcelas de 5,0 x 5,0 m para levantamento dos dados da regeneração (indivíduos com DAP inferior a 0,10 $\mathrm{m})$, divididos de acordo com as categorias de tamanho propostas por Hosokawa et al. (2008): categoria de tamanho 1 (CT1), composta por indivíduos com altura inferior a $1 \mathrm{~m}$; categoria de tamanho 2 (CT2), composta por indivíduos de 1,1 - 3,0 m de altura; e categoria de tamanho 3 (CT3), composta por indivíduos com altura superior a $3,0 \mathrm{~m}$. Também foi calculado o índice de Morisita para identificar o padrão de distribuição das espécies florestais e o índice de similaridade de Jaccard. Os dados foram processados no programa $\mathrm{R}$ versão 3.2.1.

\section{RESULTADOS}

\subsection{Atividades realizadas na Reserva Florestal de Mecubúri}

Dos 67 entrevistados, 34\% apontaram a agricultura como sendo a principal atividade desenvolvida na RFM, seguida da exploração ilegal de madeira (28\%), queimadas descontroladas (19\%), construção de habitações (14\%) e produção de carvão (5\%) (Figura 2).



Figura 2. Principais atividades desenvolvidas na RFM.

Figure 2. Main activities developed at RFM.

\subsection{Composição específica}

O levantamento realizado resultou num total de 973 indivíduos e uma riqueza de 43 espécies distribuídas em 23 famílias botânicas. Com 33 espécies, a área agrícola teve a maior riqueza, seguida da área de queimada (27 espécies). As áreas de exploração florestal e de controle tiveram apenas 22 e 21 espécies respectivamente (Tabela 1).

A família Fabaceae foi destaque nas quatro áreas, com $41 \%$ na área de queimada, $60 \%$ na área agrícola, $72,52 \%$ na área de exploração florestal e 77,5\% na área de controle.

As espécies Afzelia quanzensis, Brachystegia utilis, Burkea africana, Cussonia arborea, Julbernardia globiflora, Pericopsis angolenses, Sterculia quinquiloba e Strychnos spinosa foram as únicas observadas em todas as áreas de estudo, fato que pode ser justificado pelo fato de não sofrerem grande pressão por parte das comunidades locais.

\subsection{Distribuição diamétrica da vegetação florestal}

A distribuição diamétrica nas quatro áreas avaliadas seguiu o padrão J-invertido, característico de florestas naturais, em que a maior quantidade de indivíduos está nas classes diamétricas menores, diminuindo com o aumento das classes (Figura 3).

Tabela 1. Composição específica das áreas estudadas da RFM. Table 1. Specific composition of the studied areas at RFM.

\begin{tabular}{|c|c|c|c|c|}
\hline Espécies & $\begin{array}{c}\text { Área } \\
1\end{array}$ & $\begin{array}{c}\text { Área } \\
2\end{array}$ & $\begin{array}{c}\text { Área } \\
3\end{array}$ & $\begin{array}{c}\text { Área } \\
4\end{array}$ \\
\hline Acacia nilotica & & $\mathrm{X}$ & $\mathrm{X}$ & \\
\hline Acacia sp. & & $\mathrm{X}$ & & \\
\hline Adansonia digitata & & $\mathrm{X}$ & $\mathrm{X}$ & $\mathrm{X}$ \\
\hline Afrelia quanzensis & $\mathrm{X}$ & $\mathrm{X}$ & $\mathrm{X}$ & $\mathrm{X}$ \\
\hline Albizia adianthifolia & & $\mathrm{X}$ & & \\
\hline Albizia versicolor & & $\mathrm{X}$ & & \\
\hline Annona senegalensis & $\mathrm{X}$ & $\mathrm{X}$ & $\mathrm{X}$ & \\
\hline Baubinia petersiana & $\mathrm{X}$ & $\mathrm{X}$ & & $\mathrm{X}$ \\
\hline Brackernridgere zamguebarica & & $\mathrm{X}$ & & \\
\hline Brachystegia allonii & & $\mathrm{X}$ & $\mathrm{X}$ & $\mathrm{X}$ \\
\hline Brachystegia sp. & $\mathrm{X}$ & & & $\mathrm{X}$ \\
\hline Brachystegia utilis & $\mathrm{X}$ & $\mathrm{X}$ & $\mathrm{X}$ & $\mathrm{X}$ \\
\hline Burkea africana & $\mathrm{X}$ & $\mathrm{X}$ & $\mathrm{X}$ & $\mathrm{X}$ \\
\hline Cleistanthus holtrii & $\mathrm{X}$ & & & \\
\hline Combretum elaegnoides & $\mathrm{X}$ & $\mathrm{X}$ & & \\
\hline Combretum imberbe & $\mathrm{X}$ & $\mathrm{X}$ & & $\mathrm{X}$ \\
\hline Combretum psidiodes & $\mathrm{X}$ & & & \\
\hline Cordyla africana & & $\mathrm{X}$ & & $\mathrm{X}$ \\
\hline Cussonia arborea & $\mathrm{X}$ & $\mathrm{X}$ & $\mathrm{X}$ & $\mathrm{X}$ \\
\hline Dalbergia boehmii, & $\mathrm{X}$ & $\mathrm{X}$ & & \\
\hline Dalbergia melanoxylon & $\mathrm{X}$ & & $\mathrm{X}$ & $\mathrm{X}$ \\
\hline Dambollia xanthocarpa & & $\mathrm{X}$ & & \\
\hline Diplorbynchus condylocarpon & $\mathrm{X}$ & $\mathrm{X}$ & & $\mathrm{X}$ \\
\hline Flacourtia indica & $\mathrm{X}$ & & & \\
\hline Ficus natalensis & $\mathrm{X}$ & & $\mathrm{X}$ & $\mathrm{X}$ \\
\hline Julbernardia globiflora & $\mathrm{X}$ & $\mathrm{X}$ & $\mathrm{X}$ & $\mathrm{X}$ \\
\hline Lannea schimperi & $\mathrm{X}$ & & & $\mathrm{X}$ \\
\hline Millettia stublmannii & $\mathrm{X}$ & & $\mathrm{X}$ & $\mathrm{X}$ \\
\hline Pericorpsis angolenses & $\mathrm{X}$ & $\mathrm{X}$ & $\mathrm{X}$ & $\mathrm{X}$ \\
\hline Piliostigma thonningii & $\mathrm{X}$ & $\mathrm{X}$ & & \\
\hline Pseudolachnostylis maprouneifolia & $\mathrm{X}$ & $\mathrm{X}$ & & \\
\hline Pterocarpus angolensis & $\mathrm{X}$ & & $\mathrm{X}$ & $\mathrm{X}$ \\
\hline Sclerocarya birrea & $\mathrm{X}$ & $\mathrm{X}$ & & \\
\hline Steculia quinquiloba & $\mathrm{X}$ & $\mathrm{X}$ & $\mathrm{X}$ & $\mathrm{X}$ \\
\hline Strychnos madagascariensis & & & $\mathrm{X}$ & $\mathrm{X}$ \\
\hline Strychnos spinosa & $\mathrm{X}$ & $\mathrm{X}$ & $\mathrm{X}$ & \\
\hline Swartzia madagascariensis & & $\mathrm{X}$ & $\mathrm{X}$ & $\mathrm{X}$ \\
\hline Syzygium cordatum & & $\mathrm{X}$ & & \\
\hline Sysygium guinensis & & $\mathrm{X}$ & $\mathrm{X}$ & \\
\hline Tamarindus indica & $\mathrm{X}$ & $\mathrm{X}$ & $\mathrm{X}$ & \\
\hline Trichilia emética & & $\mathrm{X}$ & & \\
\hline Vitex doniana & & $\mathrm{X}$ & $\mathrm{X}$ & \\
\hline Vitex sp. & & $\mathrm{X}$ & $\mathrm{X}$ & \\
\hline Total & 27 & 33 & 22 & 21 \\
\hline
\end{tabular}

Ârea 1: área de queimada; Área 2: área agrícola; Área 3: área de exploração florestal; Área 4: área de controle. 


\subsection{Estrutura Horizontal}

$\mathrm{Na}$ área de queimada, a espécie Julbernardia globiflora obteve o maior peso ecológico, com o maior índice de valor de importância (IVI $=45,77)$, resultado de uma abundância (Abrel) de 11,9\%, frequência (Frel) de 9,8\% e dominância (Dorel) de 24,07\%. Seguem a Brachystegia utilis (Abrel = 13,99\%; Frel $=9,02 \%$; Dorel $=11,02 \%$; IVI $=36,03)$, a Diplorbynchus condylocarpon (Abrel $=14,8 \%$; Frel $=9,02 \%$; Dorel $=10,51 \%$; IVI $=34,33$ ) e a Burkea africana (Abrel $=$ $9,88 \%$; Frel $=5,94 \%$; Dorel $=11,48 \%$; IVI $=27,3)$.

$\mathrm{Na}$ área agrícola, Brachystegia utilis obteve o maior peso ecológico (Abrel = 18,02\%; Frel = 7,38\%; Dorel = 13,75\%; IVI $=39,15$ ) seguida de Piliostigma thonningii (Abrel $=8,15 \%$; Frel $=8,05 \%$; Dorel $=6,70 \%$; IVI $=22,9)$, Trichilia emetica $($ Abrel $=7,72 \% ;$ Frel $=6,71 \% ;$ Dorel $=6,94 \% ;$ IVI $=21,37)$ e Scleoracarya birrea (Abrel $=2,14 \%$; Frel $=2,68 \%$; Dorel $=$ $12,12 \%$; IVI $=16,94)$. Os valores obtidos indicam que a espécie é importante em termos de área basal. O número de árvores por hectare nessa área foi de 233.

Por seu turno, Millettia stublmannii obteve o maior peso ecológico na área de exploração florestal, com Abrel = $21,31 \%$, Frel $=12,24 \%$, Dorel $=24,06 \%$, IVI $=57,61)$, seguida de Cussonia arborea (Abrel $=11,48 \%$; Frel $=11,22 \%$; Dorel $=17,68 \%$; IVI $=40,38)$, Brachystegia utilis (Abrel $=$ $16,39 \%$; Frel $=11,22 \%$; Dorel $=8,25 \% ; 35$; IVI $=35,86$, Afzelia quanzensis (Abrel $=11,07 \%$; Frel $=12,24 \%$; Dorel $=$ $10,03 \%$; IVI $=33,34)$.

Já na área de controle, a espécie Brachystegia utilis teve o maior peso ecológico (Abrel $=21,34 \%$; Frel $=11,50 \%$; Dorel $=9,51 \%$; IVI $=42,35$ ), seguida de Afrelia quanzensis (Abrel = $11,85 \%$; Frel $=8,85 \%$; Dorel $=11,37 \%$; IVI $=32,07)$, Pericopsis angolenses (Abrel $=12,25 \%$; Frel $=11,5 \%$; Dorel $=$ $7,23 \%$; IVI $=30,98)$, Cussonia arborea (Abrel $=7,11 \%$; Frel $=$ $7,96 \%$; Dorel $=8,16 \%$; IVI $=23,23$ ).

O número de árvores por hectare variou de 233 (área agrícola) a 253 (área de controle).

\subsection{Regeneração natural}

Foram observados 294 indivíduos, sendo 74 pertencentes à categoria de tamanho 2 , que inclui indivíduos com altura entre 1,1 e 3 m, e 220 da categoria de tamanho 3 , que inclui indivíduos com altura superior a $3 \mathrm{~m}$ (Tabela 2). Não houve de indivíduos pertencentes à categoria de tamanho 1 (altura inferior a $1 \mathrm{~m}$ ).

Tabela 2. Regeneração natural da Reserva Florestal de Mecubúri, Moçambique.

Table 2. Natural regeneration of the Mecubúri Forest Reserve, Mozambique.

\begin{tabular}{lccc}
\hline Categoria de tamanho & CT2 & CT3 & Total \\
\hline Área agrícola & 39 & 80 & 119 \\
Área de queimada & 29 & 66 & 95 \\
Área de exploração florestal & 2 & 23 & 25 \\
Área de controle & 4 & 51 & 55 \\
\hline Total & 74 & 220 & 294 \\
\hline
\end{tabular}

\subsection{Padrão de distribuição das espécies vegetais}

Os valores do Índice de Morisita indicam que as espécies estão distribuídas de forma aleatória e em agrupamento. As espécies com baixa abundância (menos de 2 indivíduo por hectare) apresentaram índice de Morisita próximo a 1, o que indica uma distribuição espacial aleatória. Para as espécies vegetais de grande porte esse padrão aleatório, com baixa densidade de indivíduos, pode aproximar-se da sua distribuição natural na floresta, por serem espécies de maior longevidade.

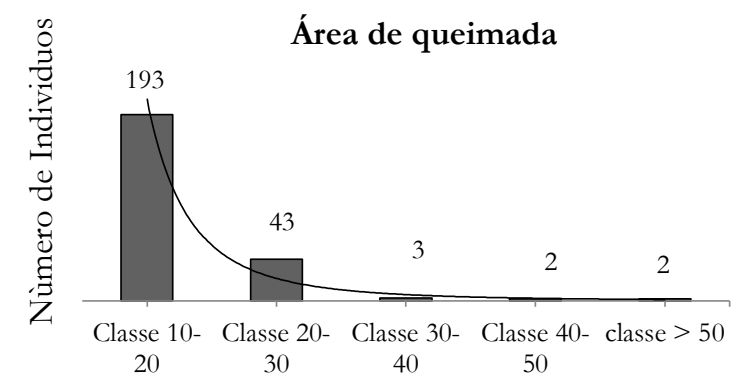

Classes diamétricas $(\mathrm{cm})$

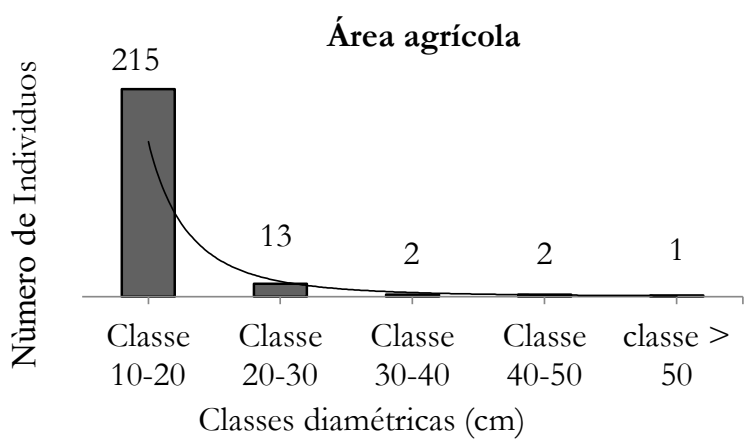

Área de exploração florestal

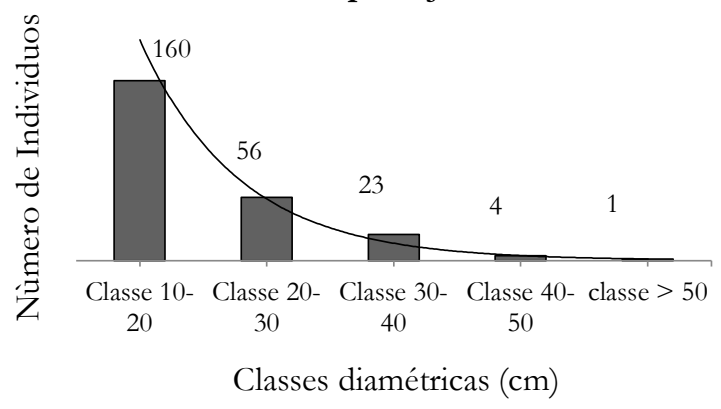

Área de controle

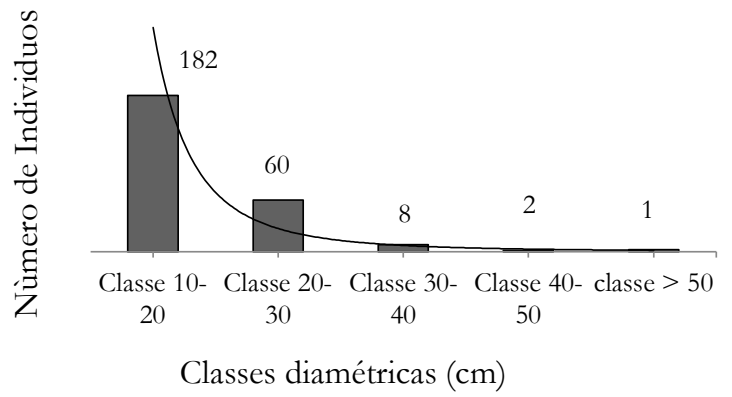

Figura 2. Distribuição dos indivíduos em classes diamétricas na RFM.

Figure 2. Distribution of individuals in diametric classes at RFM.

\subsection{Similaridade entre as áreas}

Com base no índice de Jaccard, a similaridade entre as áreas estudadas foi baixa, pois, os valores foram inferiores a 0,5 (JÚNIOR et al., 2008) (Tabela 3). 
Tabela 3. Valores do índice de similaridade de Jaccard entre as áreas estudadas da Reserva Florestal de Mecubúri, Moçambique.

Table 3. Pairwise Jaccard similarity indices between the studied areas from the Mecubúri Forest Reserve, Mozambique.

\begin{tabular}{ccccc}
\hline Área & 1 & 2 & 3 & 4 \\
\hline 1 & - & 0,37 & 0,23 & 0,13 \\
2 & & - & 0,11 & 0,15 \\
3 & & & - & 0,21 \\
4 & & & & - \\
\hline
\end{tabular}

\section{DISCUSSÃO}

As atividades identificadas nesse trabalho como sendo as mais praticadas pela população residente da RFM são, em adição à mineração, as que contribuem para a redução da cobertura florestal e degradação dos ecossistemas em Moçambique (SITOE; MAÚSSE-SITOE, 2009). De acordo com o MICOA (2007), quando estas atividades são realizadas em regiões com enormes aglomerados populacionais resultam na destruição e/ou redução da biodiversidade e dos habitats e na perda de fertilidade dos solos.

$\mathrm{Na}$ RFM, a família Fabaceae constitui a principal família, em acordo com Gomes et al. (2021), que afirma que se trata da família dominante nas florestas de Miombo, que é a vegetação característica da área de estudo. As diferenças na composição de espécies entre as áreas podem ser atribuídas à diferenças nas atividades antrópicas (queimada descontroladas, abertura de campos agrícolas e exploração florestal). As variações climáticas, edáficas, nutrientes do solo, fauna, uso passado e presente do solo, estágio sucessional da floresta, diferenças no tipo perturbação, o regime de uso florestal (concessão florestal, Reserva Florestal, zona de uso múltiplo, floresta comunitária) e outros distúrbios de origem antrópica afetam a composição de espécies de uma determinada área. A predominância de Julbernardia globiflora e as espécies do gênero Brachystegia é explicada pelo fato de serem as principais componentes da florestal de Miombo. Por seu turno, a ocorrência de espécies em uma única área, pode dever-se a sua adaptação às condições ambientais locais como saturação hídrica, teor de nutrientes e pH do solo (SILVA et al., 2009).

As principais espécies de miombo se destacaram em termos de importância ecológica nas quatro áreas da RFM. No levantamento fitossociológico realizado numa floresta de Miombo no norte de Moçambique, a Julbernardia paniculata foi a espécie ecologicamente mais importante com IVI igual a 13,3\%, seguido de Combretum adenogonium (IVI $=6,1 \%$ ), Pteleopsis myrtifolia (IVI $=5,4 \%)$, Burkea africana (IVI $=5,4 \%)$ e Brachystegia spiciformis (IVI $=5,4 \%$ ) (NANVONAMUQUITXO et al., 2019).

Os valores do número de árvores por hectare obtidos neste trabalho foram baixos quando comparado com a média obtida por Uetela (2014), que foi de 452 árvores/ha. A variação no número de árvores/ha entre as áreas pode ser justificada pelo histórico de perturbação por atividade humana, estágio sucessional, diferenças nas unidades amostrais, tipo de amostragem empregues nos estudos, e condições ambientais locais. O menor número de árvores na área agrícola revela que as atividades agrícolas são as que mais contribuem na desflorestação da RNM. Em Moçambique, todos os anos, durante a estação seca, novas áreas de floresta são cortadas para a abertura de novas áreas para a agricultura, sendo essa atividade uma das maiores ameaças à biodiversidade (MANANZE et al., 2016).
A distribuição diamétrica em j-invertido sugere que os indivíduos maiores se encontram em menor número porque são explorados. De acordo com Westphal et al. (2006), este padrão sugere a existência de um balanço entre o recrutamento e a mortalidade de indivíduos, garantindo o processo dinâmico da floresta.

A regeneração é indicativa de uma floresta em equilíbrio e em bom estado de conservação. Espécies que não apresentam regeneração correm o risco de extinção e demandam maior atenção (JUNIOR et al., 2013). No presente estudo, a quantidade reduzida de árvores nas categorias de tamanho mais baixas indica desequilíbrio na estrutura vertical da floresta, provavelmente, ocasionado por perturbações antrópicas (BRAGA et al., 2015). As espécies com menor abundância e frequência apresentaram menor regeneração nas duas categorias de tamanho por apresentarem baixa produção e dispersão de sementes (JUNIOR et al., 2013). As áreas de queimada e agrícola apresentaram maior regeneração em relação às áreas de exploração florestal e de controlo. De acordo com Chidumayo (1988), o fogo é parte integral do ecossistema de Miombo, sendo um fator chave na dinâmica da regeneração, desenvolvimento e distribuição espacial de várias espécies de fauna e flora. Porém, o impacto do fogo sobre o Miombo varia em função da época, da frequência de ocorrência e da quantidade de material combustível na floresta. No estudo de Zolho (2005) destaca-se que o efeito do fogo sobre a regeneração natural tem maior incidência sobre os principais géneros que compõem o Miombo.

Em relação ao padrão de distribuição dos indivíduos, índice de Morisita, as espécies pioneiras que crescem em florestas perturbadas ou fragmentadas geralmente encontram-se distribuídas em aglomerados, ao contrário das plantações onde as espécies distribuem-se uniformemente devido ao espaçamento definido no ato do plantio (NASCIMENTO et al., 2001). Portanto, as atividades antrópicas desenvolvidas na RFM resultaram na fragmentação da vegetação, criando conglomerados das espécies vegetais com consequência na redução da abundância. Em outro estudo em que foi analisada a diversidade florística, estrutura e distribuição espacial de espécies que ocorrem num remanescente de Floresta Ombrófila Mista, o índice de Morisita indicou que cerca de $75 \%$ das espécies apresentavam padrão de distribuição agregado (SILVESTRE, 2012).

A baixa similaridade verificada entre as áreas de estudo pode dever-se às diferenças dos componentes físicos e químicos do solo, topografia e da reduzida quantidade de espécies comuns entre as áreas. No nosso trabalho, as atividades praticadas nas diferentes áreas da RFM causam um nível de perturbação diferente. Em um estudo realizado numa área de Miombo no centro do país, foram obtidos índices de similaridade de Jaccard entre 0,15 e 0,31 para espécies pertencentes a áreas florestadas e campos agrícolas abandonados (WILLIAMS et al., 2008).

\section{CONCLUSÕES}

$\mathrm{Na}$ Reserva Florestal de Mecubúri são praticadas as seguintes atividades antrópicas que afetam a cobertura florestal: exploração florestal, queimadas descontroladas, agricultura, produção de carvão e construção de habitações. Essas atividades têm contribuído na redução da cobertura florestal e na degradação do ecossistema. Como resultado, a 
maior parte das espécies vegetais encontra-se distribuída de forma aleatória. A distribuição das árvores por classes diamétricas seguiu o padrão J-invertido, característico de florestas naturais, com o maior número de árvores na menor classe de diâmetro, reduzindo com o aumento das classes diamétricas.

As espécies Julbernardia globiflora, Diplorbynchus condylocarpon, Brachystegia utilis, Millettia stublmannii, Afželia quanzensis, Burkea africana e Cussonia arborea, são as de maior valor ecológico na área. A similaridade entre as áreas foi baixa e o maior número de árvores por hectare foi encontrado na área de controlo, sendo o menor número encontrado na área agrícola.

\section{REFERÊNCIAS}

BRAGA, E. O; SILVA, J. A. F; PANTOJA, M. V.; JARDIM, M. A. G. Florística, estrutura fitossociológica e formas de vida do estrato inferior em uma floresta de várzea Amazônica. Biota Amazónica, v. 5, n. 3, p. 59-65, 2015. DOI: http://dx.doi.org/10.18561/21795746/ biotaamazonia.v5n3p59-65

GOMES, A. L.; REVERMANN, R.; MELLER, P.; GONÇALVES, F. M. P.; AIDAR, M. P. M.; LAGES, F.; FINCKH, M. Functional traits and symbiotic associations of geoxyles and trees explain the dominance of detarioid legumes in miombo ecosystems. New Phytologist, v. 230, n. 2, p. 510-520. DOI: https://doi.org/10.1111/nph.17168

HOSOKAWA, R. T.; MOURA, J. B.; CUNHA, U. S. Introdução ao manejo e economia de florestas. Curitiba: Editora UFPR, 2008. 164 p.

JÚNIOR, E. V. F.; SOARES, T. S.; COSTA, M. F. F.; SILVA, V. S. M. Composição, diversidade e similaridade florística de uma floresta tropical semi-decídua submontana em Marcelândia - MT. Acta Amazonica, v. 38, n. 4 , p. 673-680, 2008. DOI: https://doi.org/10.1590/S0044-59672008000400010

JUNIOR, F. T. A.; FERREIRA, R. L. C.; SILVA, J. A. A.; MARANGON, L. C.; CESPEDES, G. H. G. Regeneração Natural de uma Área de Caatinga, no Sertão Pernambucano, nordeste do Brasil. Cerne, v. 19, n. 2, p. 229-235, 2013. DOI: https://doi.org/10.1590/S010477602013000200006

MAE-MOÇAMBIQUE_Ministério da Administração Estatal. Perfil de Desenvolvimento do Distrito de Mecuburi - Nampula. Maputo, 2005. 51p.

MANANZE, S. E.; SILVA, J. N.; VASCONCELOS, M. J. P. Quantificação do Desmatamento na Reserva Florestal de Mecubúri - Moçambique. Sociedade e Território, v. 28, n. 1, p. 27-47, 2016.

MARZOLI, A. Inventário Florestal Nacional. Avaliação Integrada das Florestas de Moçambique - AIFM. Maputo: Direcção Nacional de Terras e Florestas, 2007. 109p.

MICOA-MOÇAMBIQUE_Ministério para a Coordenação da Ação Ambiental. Plano de Ação para a Prevenção e Controlo às Queimadas Descontroladas 2008-2018. Maputo. 2007. 53p.

NASCIMENTO, A. R. T.; LONGHI, S. J.; BRENA, D. A. Estrutura e padrões de distribuição espacial de espécies arbóreas em uma amostra de floresta ombrófila mista em nova prata. Ciência Florestal, v. 11, n. 1, p. 109-115, 2001.

NUBE, T. G.; DOS SANTOS, A. S. J.; JUNIOR, R. T.; SILVA, I. C. Impactos socioeconômicos das plantações florestais no Niassa, Moçambique. Floresta e Ambiente, v. 23 , n. 1 , p .52-60, 2016. DOI: https://doi.org/10.1590/2179-8087.038813

SILVA, A. C.; HIGUCHI, P.; FILHO, A. T. O.; GRANATE, J. J.; MARQUES, M. Florística e estrutura da comunidade arbórea em fragmentos de floresta aluvial em São Sebastião da Bela Vista, Minas Gerais, Brasil. Revista Brasileira de Botânica, v. 32, n. 2, p. 283-297, 2009. DOI: https://doi.org/10.1590/S010084042009000200008

SITOE, A.; MAÚSSE-SITOE, S. Construindo parcerias florestais: Potencial das reservas florestais na redução do desmatamento com participação das comunidades locais. Maputo: ICPF, 2009. 22p.

SOUZA, P. A. de; VENTURIN, N.; MACEDO, R. L. G.; ALVARENGA, M. I. N.; SILVA V. F. da. Estabelecimento de espécies arbóreas em recuperação de área degradada pela extração de areia. Cerne, v. 7, n. 2, p. 43-52, 2001.

UETELA, A. E. Dinâmica e prognose de produção de uma floresta de Miombo utilizando matriz de transição. 2014. Dissertação (Mestrado em Ciências Florestais). Universidade Federal do Paraná, Curitiba. 2014. 90p. Disponível em $<$ http://www.floresta.ufpr.br/posgraduacao/defesas/pdf_ms/2014/d687_0180-M.pdf>. Acesso em: 23 set 2020.

$\mathrm{ZOLHO}, \mathrm{R}$. Effect of fire frequency on the regeneration of Miombo woodland in Nhambita, Mozambique. Tese de mestrado. University of Edinburgh. 2005. 71p. Dissertação (Mestrado em Ciências). Universidade de Edinburgh. Disponível em < https://www.biofund.org.mz/biblioteca_virtual/effectof-fire-frequency-on-the-regeneration-of-miombowoodland-in-nhambita-mozambique/>. Acesso em: 26 jun 2020.

NANVONAMUQUITXO, S. J. A.; MACUEIA, F. B. E. D.; CARAVELA, M. I. Estrutura e diversidade de uma floresta de Miombo em Taratibu, Norte de Moçambique. Nativa, v. 7, n. 6, p. 778-783. 2019. DOI: http://dx.doi.org/10.31413/nativa.v7i6.7198

CHIDUMAYO, E. N. A re-assessment of effects of fire on miombo regeneration in the Zambian Copperbelt. Journal of Tropical Ecology, v. 4, n. 4, p. 361-372, 1988.

WILLIAMS, M.; RYAN, C. M.; REES, R. M.; SAMBANE, E.; FERNANDO, J.; GRACE, J. Carbon sequestration and biodiversity of re-growing miombo woodlands in Mozambique. Forest Ecology and Management, v. 254, n. 2, p. 145-155, 2008. DOI: https://doi.org/10.1016/j.foreco.2007.07.033.

SILVESTRE, R.; KOEHLER, H. S.; MACHADO, S. A.; BALBINOT, R.; WATZLAWICK, L. F. Florística, estrutura e distribuição espacial de espécies ocorrentes em um remanescente de floresta ombrófila mista. Ambiência, v. 8, n .2, p. 259-274, 2012. 